\title{
Numerical Simulations of Stick Percolation: Application to the Study of Structured Magnetorheological Elastomers
}

\author{
J. L. Mietta, ${ }^{\dagger}$ R. M. Negri, ${ }^{\dagger}$ and P. I. Tamborenea ${ }^{* \dagger}$ \\ ${ }^{\dagger}$ Instituto de Química-Física de Materiales, Ambiente y Energía (INQUIMAE), Facultad de Ciencias Exactas y Naturales, Universidad \\ de Buenos Aires, Ciudad Universitaria, Pabellón II, Ciudad de Buenos Aires, Argentina \\ ${ }^{\ddagger}$ Departamento de Física and Instituto de Física de Buenos Aires (IFIBA), Facultad de Ciencias Exactas y Naturales, Universidad de \\ Buenos Aires, Ciudad Universitaria, Pabellón I, Ciudad de Buenos Aires, Argentina
}

ABSTRACT: In this article we explore how structural parameters of composites filled with one-dimensional, electrically conducting elements (such as sticks, needles, chains, or rods) affect the percolation properties of the system. To this end, we perform Monte Carlo simulations of asymmetric two-dimensional stick systems with anisotropic alignments. We compute the percolation probability functions in the direction of preferential orientation of the percolating objects and in the orthogonal direction, as functions of the experimental structural parameters. Among these, we considered the average length of the sticks, the standard deviation of the length distribution, and the standard deviation of the angular distribution. We developed a computer algorithm capable of reproducing and verifying known theoretical results for isotropic networks and which allows us to go beyond and study anisotropic
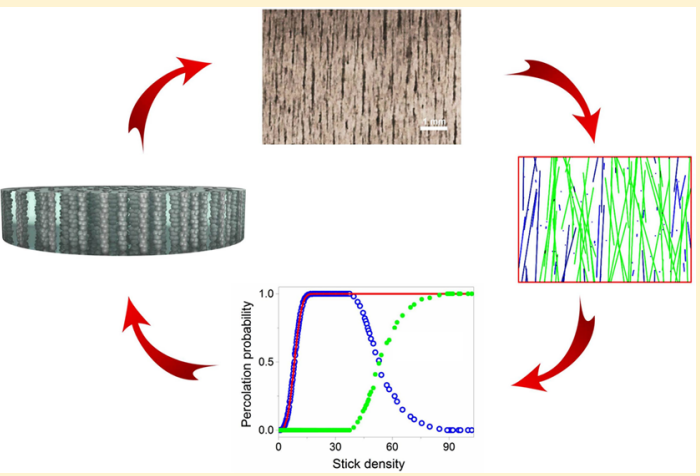
systems of experimental interest. Our research shows that the total electrical anisotropy, considered as a direct consequence of the percolation anisotropy, depends mainly on the standard deviation of the angular distribution and on the average length of the sticks. A conclusion of practical interest is that we find that there is a wide and well-defined range of values for the mentioned parameters for which it is possible to obtain reliable anisotropic percolation under relatively accessible experimental conditions when considering composites formed by dispersions of sticks, oriented in elastomeric matrices.

\section{INTRODUCTION AND EXPERIMENTAL MOTIVATION}

Recently, vigorous interest has arisen in percolating networks built out of nano- and microdimensional objects (percolating objects), such as nanotubes and nanowires for various applications such as thin film transistors, ${ }^{1,2}$ flexible microelectronics, ${ }^{3}$ microelectromechanical systems (MEMS), ${ }^{4-6}$ chemical sensors, ${ }^{7}$ and construction of transparent electrodes for optoelectronic and photovoltaic devices. ${ }^{8,9}$ In particular, if these objects are used as fillers dispersed in an elastomeric polymer and then oriented inside the organic matrix, there is an anisotropic internal structure to the material. ${ }^{10,11}$ This anisotropic structure can be obtained in practice following different methods, like, for example, orienting the filler particles by means of an external field (electric or magnetic), mechanically squeezing a composite material, etc. A particular case is given by magnetorheological materials, whose mechanical properties can be modified by externally applied magnetic fields. An important example of magnetorheological materials are composites formed by dispersing magnetic filler particles into an elastomer polymeric matrix and then orienting the particles. These materials are referred to as magnetorheological elastomers (MREs). If, additionally, the filler particles are electrically conducting, the MRE may also be a conductor depending on the properties of the filler and matrix materials and on the conditions of synthesis. A simple procedure to obtain MREs (in film or bulk form) consists of curing the filler-elastomer composite in the presence of a uniform magnetic field, which induces agglomerations of the filler particles into chainlike structures (needles) aligned in the direction of the magnetic field. ${ }^{4,6,12-14}$

A desirable property in an electrically conducting MRE is its electrical anisotropy, i.e., its ability to conduct an electrical current preferentially in a special direction. Although we will not tackle here the full problem of the relation between percolation and electrical conductivity, for our current purposes it will suffice to use the fact that there will be total electrical anisotropy (TEA) in the MRE, i.e., conduction in only a chosen direction, if there are percolating paths formed only in that chosen direction. This direction is given by the preferred orientation of the needles, which coincides with the direction of magnetic field applied during the curing of the material. For example, TEA is a crucial property in devices like extended pressure mapping sensors, ${ }^{6,12}$ Zebra-like connectors for parallel flip-chip connections, ${ }^{4}$ and others. If the experimental variables are not properly set during the fabrication of the MRE, a

Received: April 29, 2014

Revised: July 25, 2014

Published: August 7, 2014 
material devoid of anisotropy (or with very low anisotropy) might be obtained, ${ }^{4,12,15}$ which would be unsuitable for these types of applications.

The microscopic structure of an MRE film filled with randomly distributed magnetic sticks can be characterized by three parameters: the average length of the sticks, $\langle l\rangle$, the standard deviation of the length distribution, $\sigma_{v}$ and the standard deviation of the angular distribution, $\sigma_{\theta}$ (around a chosen direction). As will be seen later, in the experimental samples these parameters correspond to a Gaussian distribution for the angle $\theta$ and a log-normal distribution for the length $l$. These parameters can be set experimentally by changing the intensity of the magnetic field during the curing, the exposure time to the magnetic field before starting the thermal curing, the viscosity of the matrix, the amount of filler, the magnetic properties of the filler, etc. Two of us have performed several experimental studies of these systems, ${ }^{4,6,12,13}$ and now we wish to understand how their structural parameters affect the probability of obtaining systems with TEA. Nevertheless, it can be expected that the results of our modeling will also apply to other composite materials with similar internal structure.

In magnetorheological elastomers, micro- or nanoscopic objects (called alternatively chains, needles, or sticks) are formed by magneto-piezo-electric manipulation under appropriate experimental conditions at various stages of the synthesis process. ${ }^{4,6,12,15}$ These sticks formed by the filler material can be considered as quasi-one-dimensional objects, so that the nanostructured MRE as a whole can be analyzed in terms of networks of percolating sticks. Percolation can be studied either in bulk (3D) or planar (2D) geometry. For our purposes (i.e., studying the TEA) both situations provide useful models, and we will therefore consider the latter, which allows us to explore numerically larger systems. In rectangular or square MRE films, by definition there is a spanning cluster if there is at least one connecting path between two opposing electrical contacts (located on opposite edges of the film) formed by intersecting sticks. In order to relate electrical conduction to percolation concepts, here we adopt the criterion that there is TEA if and only if there is a spanning cluster in one direction and not in the other. Thus, we adopt here the well-known two-dimensional model of percolating sticks, which is an example of a continuous percolation model ${ }^{16-23}$ in order to study the dependence of the TEA on the structural parameters $\sigma_{\theta},\langle l\rangle$, and $\sigma_{l}$. We follow the approach of studying the percolation probability functions in the direction of application of the curing magnetic field and in the orthogonal direction, as functions of the experimental parameters $\sigma_{\theta}, \sigma_{l}$ and $\langle l\rangle$. For this, we have developed a computer algorithm that is able to reproduce and verify known theoretical results for isotropic networks and which allows us to go beyond and study anisotropic systems of experimental interest.

Most related studies in the literature deal with square systems, with uniform stick length and isotropic angular distributions, ${ }^{2,16,24}$ but the issue of anisotropy has also attracted a great deal of attention in recent years. Du, Fischer, and Winey ${ }^{10}$ studied the effect of the alignment of carbon nanotubes on the percolation in their polymer/nanotube composites. Their experiments and simulations introduce the interesting point that angular dispersion also can produce (besides stick density) a percolation transition with critical behavior. Our work is different from or goes beyond theirs in a number of ways. Importantly, we concentrate on TEA, monitored via the percolation probability in only one direction in rectangular systems. Furthermore, we gave the sticks realistic log-normal distributions for their lengths and Gaussian distributions for their azimuthal angles, and we considered rectangular samples. These conditions were dictated by our own experimental MRE samples. Instead, Du et al. ${ }^{10}$ considered in their simulations monodispersed (fixed length) sticks, their angular dispersion is uniform within an angle interval (a "wedge"), and they focused on square systems. More recently, Zheng et al. ${ }^{25}$ reported on numerical simulations of threedimensional percolation of cylindrical rods with both isotropic and anisotropic angular distributions. They study the effect of the aspect ratio of the percolating objects and consider monodisperse (identical) rods. For anisotropic systems they consider a particular type of angular distribution produced by the interaction with a flow. White et al. ${ }^{26}$ consider the issue of alignment in percolation of soft-core cylindrical particles, employing a continuous angular distribution, as a function of which they study the percolation transition in $3 \mathrm{D}$ square systems. Besides the 3D nature of the system and the different choice of angular distribution, a major difference with our present study is again the fact that they do not focus on TEA but rather on general percolation, like it is done also in ref 25 . The horizontal-only percolation transition that we focus on here has recently been considered, for percolation of sticks, among other objects, by Mertens and Moore ${ }^{27}$ (see also ref 28 ). However, their main goal is to introduce a new simulation technique for continuum percolation models, and for the case of stick percolation they only compute the wrapping percolation threshold for square systems with isotropically distributed sticks. In turn, in the present work we study stick percolation in rectangular systems with log-normal length distributions and Gaussian angular distributions and explore the full range of parameters of those two distributions in search of TEA. As a result of our simulations, we are able to specify the special "safety zones" of parameter space where that goal is achieved.

\section{ALGORITHM OF STICK-PERCOLATION SIMULATIONS}

We study two-dimensional stick percolation by means of computer simulations. The percolation probabilities are obtained by repeating a large number of times a percolation experiment (a "realization"). Each experiment consists of, starting with an empty rectangular ( sides $L_{x}, L_{y}$, aspect ratio $r=$ $L_{x} / L_{y}$ ) or square (side $L=L_{x}=L_{y}$ ) box, adding a desired number $\boldsymbol{N}$ of sticks of length $\boldsymbol{l}$ (either a fixed length or statistically distributed values), and determining whether a spanning cluster exists between the vertical and/or horizontal faces of the box. The orientation of a stick is given by an angle $\theta$ with respect to a horizontal axis, also generated randomly, with in general $-\pi \leq \theta \leq \pi$. Depending on the characteristics of the physical system that one wishes to simulate, different statistical distributions for the lengths $l$ and the angles $\theta$ can be adopted. For example, the simplest and most usually studied model is the one with a uniform length for all sticks and isotropic angular distribution. Once the sticks of a given realization (our "percolating objects") have been generated, we need to first determine which sticks intersect. Let $A_{i}$ and $B_{i}$, for $i=1, \ldots, N$, denote the end points of the sticks. For algorithmic purposes, the sticks can be seen as vectors, ${\overrightarrow{A_{i}}}_{i}$. Consider a subsystem consisting of only two sticks, $\overrightarrow{A_{1} B_{1}}$ and $\overrightarrow{A_{2} B_{2}}$. It can be 
demonstrated that they intersect if the following conditions are simultaneously satisfied ${ }^{29}$

$$
\begin{aligned}
& \left(\overrightarrow{A_{1} B_{1}} \times \overrightarrow{A_{1} A_{2}}\right) \cdot\left(\overrightarrow{A_{1} B_{1}} \times \overrightarrow{A_{1} A_{2}}\right) \leq 0 \\
& \left(\overrightarrow{A_{2} B_{2}} \times \overrightarrow{A_{2} B_{1}}\right) \cdot\left(\overrightarrow{A_{2} B_{2}} \times \overrightarrow{A_{2} B_{1}}\right) \leq 0
\end{aligned}
$$

The intersection pattern of the $N$ sticks is explored pairwise with these conditions, and an $N \times N$ intersection matrix $\mathrm{J}$ is formed such that $J_{i j}=1$ if the sticks $i$ and $j$ intersect, and $J_{i j}=0$ if they do not. The matrix $\mathbf{J}$ is then used as the matrix associated with an intersection graph, and a Deep First Search algorithm ${ }^{30}$ is implemented to evaluate the presence of spanning clusters connecting the opposite edges of the system. If out of $n$ realizations of the system $k$ of them possess at least one spanning cluster, then $k / n$ is an unbiased estimator of the percolation probability (probability that a system has at least one cluster, referred here as $\boldsymbol{P}$ ), i.e., $k / n \rightarrow \boldsymbol{P}$ with $n \rightarrow \infty$. $X \equiv$ $k / n$ is a binomially distributed random variable with mean $E[X]$ $=\boldsymbol{P}$ and variance $\operatorname{Var}[X]=[\boldsymbol{P} \times(1-\boldsymbol{P})] / n^{31}$ Then, for acceptable accuracy (estimator error lower than $2 \%$ ), at least $10^{3}$ Monte Carlo realizations were performed, yielding comparable or even better statistics than those found in recent studies. $^{2,24} \mathrm{We}$ implemented our algorithm in a computer program written in SAGE.

Figure 1 shows three examples of random stick systems in a square two-dimensional box of side $L=5$ with isotropic angular distribution and fixed stick length $l=1$ (lengths expressed in the same arbitrary units), for different stick densities, $\Phi=N / L^{2}$, especially chosen in order to display the percolation behavior, well below, slightly above, and well above the percolation critical density. For the low density there are no spanning clusters. For the described system, for the intermediate density there is one spanning cluster which contains about $60 \%$ of the sticks, and for the high density most of the sticks participate in the spanning cluster.

\section{FINITE-SIZE SCALING FOR SQUARE ISOTROPIC SYSTEMS}

In order to validate our computer-simulation techniques, we first reproduce some key scaling results available in the literature on stick percolation for square systems. Let us consider square systems (aspect ratio $r=1$ ) of side $L$ with $N$ sticks of fixed length $l$. The stick density is thus $\Phi=N / L^{2}$. The percolation probability $\boldsymbol{P}_{L, l}(\Phi)$ (i.e., the probability that there is at least one spanning cluster in the horizontal direction regardless of what happens in the vertical direction, to be called $\boldsymbol{P}_{L, l}^{\mathrm{H}}$ later on) is shown in Figure $2 \mathrm{a}$ as a function of $\Phi$ for different values of $L$ and $l$. The percolation probability is estimated for each density by running $n>1000$ realizations.

For finite systems, we wish to obtain the critical density $\langle\Phi\rangle_{L, l}$ which, according to the Renormalization Group (RG) theory for square systems $(r=1)$, scales with the system size $L$ as ${ }^{17,19,20,27,32}$

$$
\langle\Phi\rangle_{L, l}=\Phi_{\infty, l}+a_{l} L^{-1 / \nu-\vartheta}
$$

The universal scaling exponent is $\nu=4 / 3$ for all twodimensional percolation systems including lattice and continuum percolation (in particular, 2D random sticks systems). Recently, it has been found that in random stick-percolation square systems $(r=1)$ the nonanalytical correction given by the exponent $\vartheta$ takes the value $0.83 \pm 0.02,{ }^{19}$ consistent with previously published results of $72 / 91,{ }^{33} 0.85,{ }^{34}$ and $0.90 \pm$
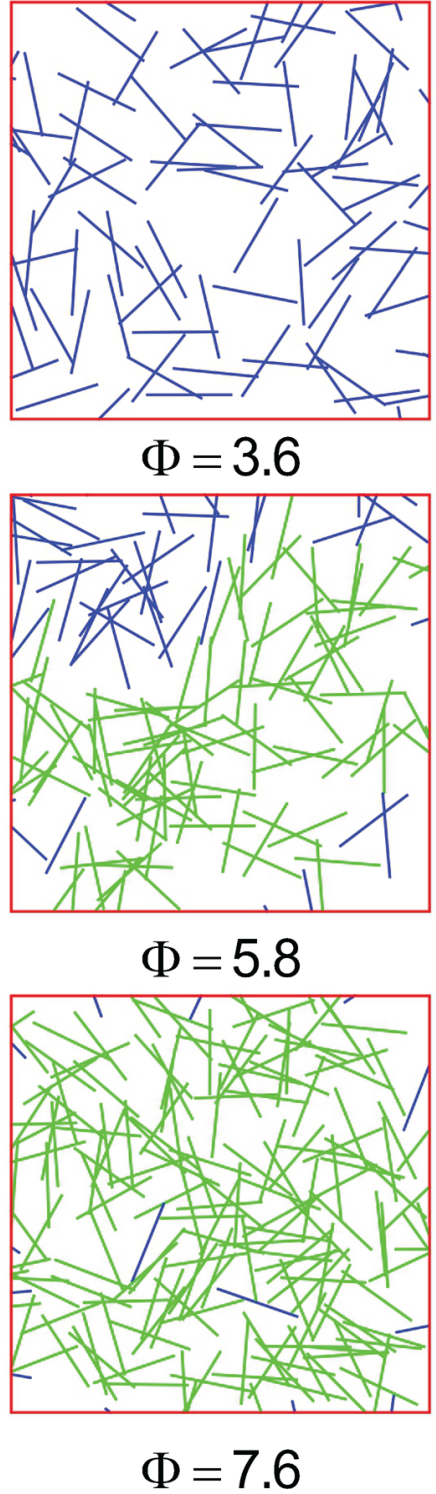

Figure 1. Examples of random stick systems in a square twodimensional box of side $L=5$ with isotropic angular distribution and stick length $l=1$ (all lengths are expressed in the same arbitrary units), for different stick densities. The green (color online) sticks belong to a horizontal spanning cluster and the blue ones do not.

$0.02,^{35}$ while in rectangular systems $(r \neq 1) \vartheta=0 .{ }^{19}$ The probability distribution function

$$
\Gamma_{L, l}(\Phi)=\frac{\partial \boldsymbol{P}_{L, l}(\Phi)}{\partial \Phi}
$$

is usually assumed to be Gaussian. ${ }^{2,36}$ Therefore, from curves like the ones shown in Figure 2a one can extract the sizedependent critical percolation density $\langle\Phi\rangle_{L, l}$ and the standard deviation of the distribution function $\Delta_{L, l}$ by fitting the simulated values of $\boldsymbol{P}_{L, l}$ to an error-function $\operatorname{erf}(x)$ according to

$$
\boldsymbol{P}_{L, \boldsymbol{l}}(\Phi)=\frac{1}{2}\left[1+\operatorname{erf}\left(\frac{\Phi-\langle\Phi\rangle_{L, \boldsymbol{l}}}{\Delta_{L, l}}\right)\right]
$$

where $\operatorname{erf}(x)=(2 / \sqrt{ } \pi) \int_{0}^{x} \mathrm{e}^{-t^{2}} \mathrm{~d} t$. The data shown in Figure 2a are known not to be given exactly by error functions in their tails, but rather by stretched exponentials. ${ }^{28}$ However, as can be 

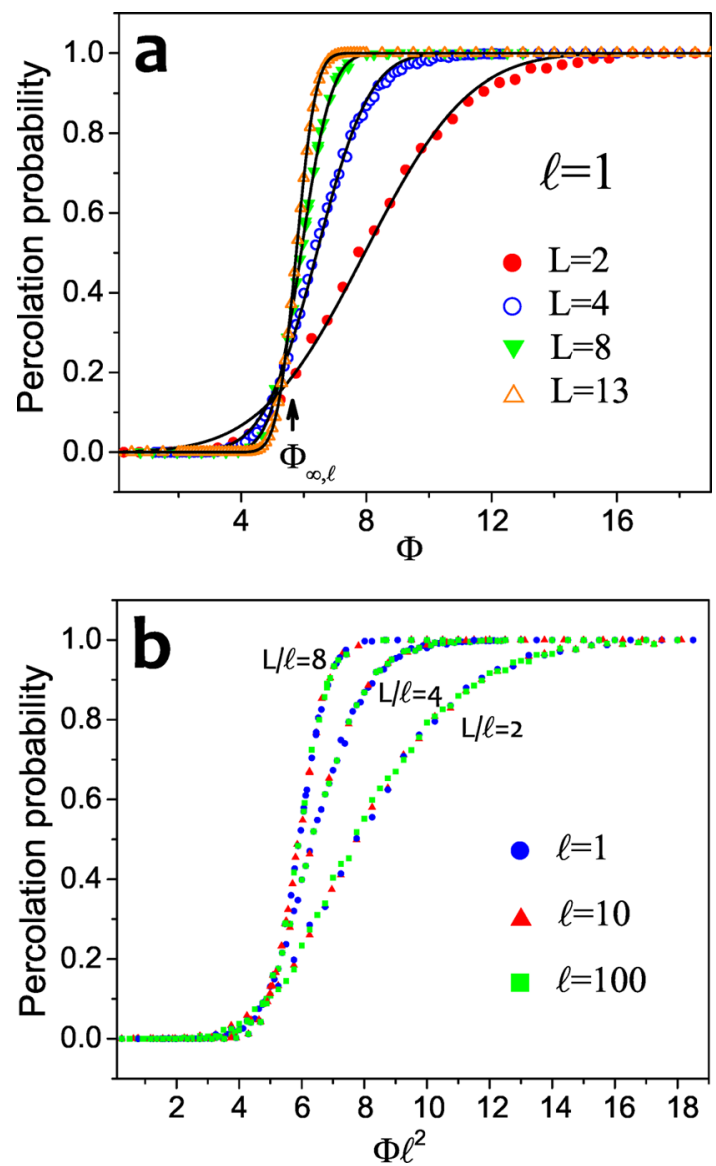

Figure 2. Percolation probability $\boldsymbol{P}_{L, l}$ for square systems of percolating sticks with isotropic angular distribution. (a) Stick length $l=1$; the continuous lines are fits to error functions, eq 4. (b) Various values of $L$ and $l$, displaying the collapse of data when the percolation probability is plotted against $\Phi l^{2}$.

seen in the figure, we obtain excellent fits $\left(R^{2} \geq 0.9992\right)$, validating for all practical purposes the assumption of Gaussian distribution that leads to the error functions displayed as solid lines in Figure 2a.

A quick and rough estimate of the asymptotic critical percolation density $\Phi_{L \rightarrow \infty, l}$ (or $\Phi_{\infty, l}$ ) can be obtained by simulating a system with large $L$ and employing $\boldsymbol{P}_{L, l}\left(\langle\Phi\rangle_{L, l}\right)=$ $1 / 2$. Indeed, the curve with $L=13$ and $l=1$ in Figure 2 a shows a fairly sharp transition at the critical stick density $\langle\Phi\rangle_{L=13, l=1}$, which is in fairly good agreement with the accepted value of $\Phi_{\infty, l=1}=5.6372858(6) .^{29}$

As expected, in Figure 2a the percolation transition becomes less sharp for diminishing system size $L$ (according to the RG theory the standard deviation scales as $\Delta_{L, l} \propto L^{-1 / \nu}$ for isotropic square systems), and at the same time the critical density $\langle\Phi\rangle_{L, l}$ shifts toward higher values (eq 2). Our simulations accurately reproduce the expected scaling laws for the critical density and the standard deviation, as seen in Figure 3 for stick length $l=1$. From these curves we obtain $\vartheta=0.83 \pm 0.04$ and $\nu=1: 33 \pm$ 0.03 in agreement with previously reported values. ${ }^{19,33-35}$

The dependence of the percolation probability on the stick length, $l$, can be understood thanks to the RG theory, which suggests that there is a relationship between $\Phi_{\infty, l}$ and $l$ given by the effective area associated with each percolating element. Associating an effective self-area $l^{2}$ to each stick, one obtains the relationship ${ }^{17,27}$

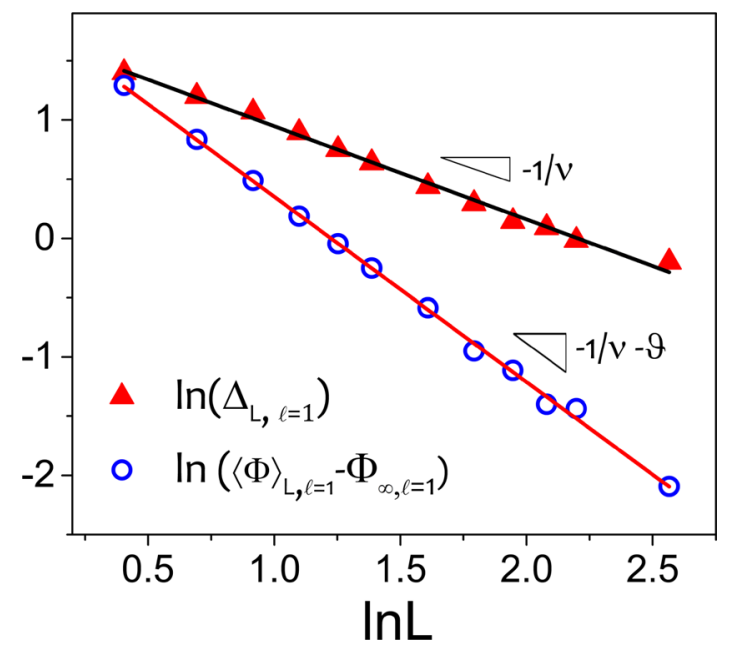

Figure 3. Critical percolation density $\langle\Phi\rangle_{L, l}$ and standard deviation $\Delta_{L, l}$ versus system size $L$ for square systems with stick length $l=1$ (measured in the same units as $L$ ).

$$
\Phi_{\infty, l} l^{2}=\Phi_{\infty, l=1}=5.6372858(6)
$$

Plotting the percolation probability for different values of $l$ as a function of $\Phi l^{2}$ (instead of doing it simply as a function of $\Phi$ ) shows that the most relevant quantity is the ratio $L / l$ rather than $L$ and $l$ independently. In simple terms, this indicates that what matters in the definition of the density of sticks is the system size measured in units of the typical size of the percolating objects. In Figure $2 \mathrm{~b}$ we plot the percolation probability for three values of $l=1,10,100$ and corresponding values of the system size $L$ such that $L / l=2,4,8$. The excellent collapse of curves with equal ratio $L / l$ ratifies the validity of the RG analysis leading to eq 5 .

In section 5 we will study anisotropic systems which require, to be described from the point of view of percolation theory, the introduction of additional percolation probabilities. We introduce their definitions here, in order to use them in a last check of known results for square systems. Let us denote the probabilities of having different types of spanning clusters as follows: horizontally $\boldsymbol{P}^{\mathrm{H}}$, vertically $\boldsymbol{P}^{\mathrm{V}}$, only horizontally $\boldsymbol{P}^{\mathrm{HX}}$, only vertically $\boldsymbol{P}^{\mathrm{VX}}$, on either direction $\boldsymbol{P}^{\mathrm{U}}$, and on both directions $\boldsymbol{P}^{\mathrm{HV}}$. These probabilities are not independent of each other, as they satisfy $\boldsymbol{P}^{\mathrm{U}}=\boldsymbol{P}^{\mathrm{H}}+\boldsymbol{P}^{\mathrm{V}}-\boldsymbol{P}^{\mathrm{HV}}$ and $\boldsymbol{P}^{\mathrm{U}}=\boldsymbol{P}^{\mathrm{HX}}+\boldsymbol{P}^{\mathrm{VX}}$ $+\boldsymbol{P}^{\mathrm{HV}} \cdot{ }^{27,28}$ In Figure 4 we show the probabilities $\boldsymbol{P}^{\mathrm{H}}, \boldsymbol{P}^{\mathrm{HV}}, \boldsymbol{P}^{\mathrm{HX}}$, and $P^{\mathrm{U}}$ at the critical percolation density $\Phi_{\infty, l}=5.6372858$ versus the system size $L$ for square systems with uniform stick length $(l=1)$ and isotropic stick distribution. The asymptotic regime $(L \rightarrow \infty)$ is clearly seen since it is reached at $L \approx 30$, and we show results up to $L=100$. We verify that our asymptotic values (which we take to be the ones for $L=100$ ) agree very well with those found in the literature. For $P^{\mathrm{H}}$ we obtain $0.51 \pm 0.03$ while the exact value is $0.5 .^{19,37-41}$ For $P^{\mathrm{HV}}$ we get $0.33 \pm 0.03$ while the also exact value is 0.322120 $45 .^{38-41}$ For $\boldsymbol{P}^{\mathrm{U}}$ we have $0.69 \pm 0.03$ while 0.67787955 is the expected value from the formula $P^{\mathrm{U}}=\boldsymbol{P}^{\mathrm{H}}+\boldsymbol{P}^{\mathrm{V}}-\boldsymbol{P}^{\mathrm{HV}}$ (note that for square and isotropic systems $\boldsymbol{P}^{\mathrm{H}}=\boldsymbol{P}^{\mathrm{V}}$ ). Finally, for $\boldsymbol{P}^{\mathrm{HX}}$ we obtain $0.18 \pm 0.02$ while 0.17787955 is obtained with $P^{\mathrm{HX}}$ $=\left(P^{\mathrm{U}}-\boldsymbol{P}^{\mathrm{HV}}\right) / 2$. To the best of our knowledge, this is the first numerical validation in stick percolation of the existing theoretical predictions for the different probabilities $\boldsymbol{P}^{\mathrm{HX}}, \boldsymbol{P}^{\mathrm{HV}}$, and $P^{\mathrm{U}}$ in the limit of large system size at the critical percolation density. 


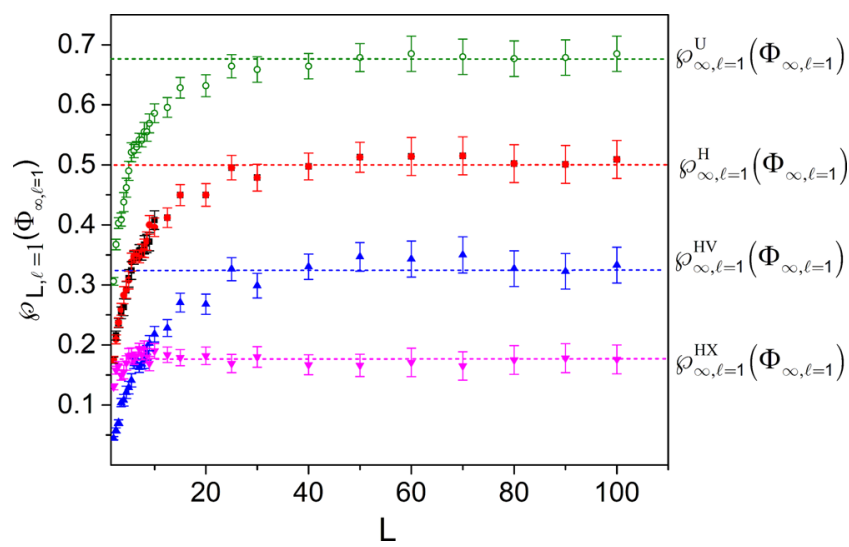

Figure 4. System-size dependence of the different percolation probabilities at the critical percolation density, $\Phi_{\infty, l=1}$. The asymptotic $(L \rightarrow \infty)$ behavior is clearly seen. The results are for square systems with uniform stick length $(l=1)$ and isotropic stick distribution.

\section{SYNTHESIS AND EXPERIMENTAL MORPHOLOGICAL CHARACTERIZATION OF MRES}

The elastomeric material that we studied is comprised of a poly(dimethylsiloxane) (PDMS) polymer matrix and of percolating chains consisting of hybrid magnetite-silver microparticles. These microparticles have an internal structure consisting of clusters of magnetite nanoparticles covered with metallic silver. Using the hybrid filler material described above allows us to obtain a current-conducting magnetorheological material in superparamagnetic state (because magnetite nanoparticles, due to their small diameter, are in a superparamagnetic state at temperatures higher than their blocking temperature, $T_{\mathrm{B}}=179 \mathrm{~K}$ ). The electrical conductivity is not affected by oxidation, given by the chemical fastness of silver metal. Finally, the use of PDMS as polymer matrix increases the chemical resistance of the composite against various chemical agents such as aromatic solvents, halogenated aliphatic solvents, aliphatic alcohols, and concentrated salt solutions. ${ }^{4,12}$

The preparation method used to obtain the structured MRE composite with magnetic $\mathrm{Fe}_{3} \mathrm{O}_{4}$ silver-covered microparticles in PDMS (referred to as PDMS- $\mathrm{Fe}_{3} \mathrm{O}_{4} @ \mathrm{Ag}$ ) was described in detail in previous works ${ }^{4,12}$ and is briefly described here. First, $\mathrm{Fe}_{3} \mathrm{O}_{4}$ superparamagnetic nanoparticles (NPs) were synthesized by the chemical coprecipitation method where a solution mixture (2:1) of $\mathrm{FeCl}_{3} \cdot 6 \mathrm{H}_{2} \mathrm{O}$ and $\mathrm{FeCl}_{2} \cdot 4 \mathrm{H}_{2} \mathrm{O}$ in chlorhydric acid was added drop-by-drop to a solution of $\mathrm{NaOH}\left(60{ }^{\circ} \mathrm{C}\right.$, $\mathrm{pH}=14$ ), under nitrogen atmosphere and high-speed stirring. The obtained nanocrystals were separated by repeated centrifugation and washing cycles and then dried in a vacuum oven at $40{ }^{\circ} \mathrm{C}$ during $24 \mathrm{~h}$. The obtained dark brown NPs show a size distribution (determined by TEM images) with maximum at $13 \mathrm{~nm}$ in the log-normal distribution of diameters, which is in excellent agreement with the size of the crystallite domains calculated using the Debye-Scherrer relation from $\mathrm{X}$-ray difractograms (XRD), $(14 \pm 2) \mathrm{nm} .{ }^{4,12,42,43}$

In a second step, the $\mathrm{Fe}_{3} \mathrm{O}_{4} \mathrm{NPs}$ were covered with silver in order to obtain electrically conductive and superparamagnetic particles. For that, aqueous dispersions of $\mathrm{Ag}\left(\mathrm{NH}_{3}\right)_{2}^{+}$and $\mathrm{Fe}_{3} \mathrm{O}_{4}$ NPs in a 10:1 molar ratio were sonicated for $30 \mathrm{~min}$ at room temperature. Then the system was heated in a water bath at 40 ${ }^{\circ} \mathrm{C}$ for $20 \mathrm{~min}$ with slow stirring. In the following step, $0.4 \mathrm{M}$ glucose monohydrate solution was added drop-by-drop to the $\mathrm{Fe}_{3} \mathrm{O}_{4}-\mathrm{Ag}^{+}$suspension. Stirring was continued for $1 \mathrm{~h}$. This synthesis protocol promotes the reduction of $\mathrm{Ag}(\mathrm{I})$ ions adsorbed onto $\mathrm{Fe}_{3} \mathrm{O}_{4}$ particles. The magnetite-silver particles were separated out from the solution by magnetization and then by centrifugation. After the particles were separated, the decanted supernatant liquid was fully transparent. The obtained system (referred to here as $\mathrm{Fe}_{3} \mathrm{O}_{4} @ \mathrm{Ag}$ ) is actually formed by microparticles whose internal structure consists of several $\mathrm{Fe}_{3} \mathrm{O}_{4}$ nanoparticles clusters covered by metallic silver grouped together. For the $\mathrm{Fe}_{3} \mathrm{O}_{4} @ \mathrm{Ag}$ microparticles (MPs) the maximum of the diameter distribution is at $1.3 \mu \mathrm{m}$ (determined by SEM and TEM images). For comparison purposes, silver particles (reddish orange) were produced in a separate batch using the same experimental conditions for each set.

Finally poly(dimethylsiloxane) (PDMS) base and curing agent, referred to as PDMS from now on (Sylgard 184, Dow Corning), were mixed in proportions of 10:1 (w/w) at room temperature and then loaded with the magnetic $\mathrm{Fe}_{3} \mathrm{O}_{4}$ silvercovered microparticles. The amounts of PDMS and fillers were weighed during mixing on an analytical balance, homogenized, and placed at room temperature in a vacuum oven for about $2 \mathrm{~h}$ until the complete absence of any air bubble is achieved. Specifically, composite material with $5 \%$ w/w of $\mathrm{Fe}_{3} \mathrm{O}_{4} @ \mathrm{Ag}$ was prepared. The still fluid samples were incorporated into a specially designed cylindrical mold $(1 \mathrm{~cm}$ diameter by $1.5 \mathrm{~cm}$ thickness) and placed in between the magnetic poles of a Varian Low Impedance Electromagnet (model V3703), which provides highly homogeneous steady magnetic fields. The mold was rotated at $30 \mathrm{rpm}$ to preclude sedimentation and heated at $(75 \pm 5){ }^{\circ} \mathrm{C}$ in the presence of a uniform magnetic field $\left(H_{\text {curing }}=0.35 \mathrm{~T}\right)$ over $3 \mathrm{~h}$ to obtain the cured material. The polymeric matrix is formed by a tridimensional cross-linked siloxane oligomers network with $\mathrm{Si}-\mathrm{CH}_{2}-\mathrm{CH}_{2}-\mathrm{Si}$ linkages. $^{44,45}$ Slices of the cured composites were held in an adhoc sample-holder and cut using a sharp scalpel, which were used for the morphological (SEM and optical microscopy analysis) and electrical characterization of material.

All fabricated composites obtained following the procedure described above displayed total electrical anisotropy, showing significant electrical conductivity only in the direction of application of the magnetic field during curing (which coincides with the direction of $\mathrm{Fe}_{3} \mathrm{O}_{4} @ \mathrm{Ag}$ chains). For these MRE materials, electrical resistivity values of $\rho_{\|}=4 \Omega \times \mathrm{cm}$ and $\rho_{\perp}=$ $60 \mathrm{M} \Omega \times \mathrm{cm}$ were obtained, where $\|$ and $\perp$ indicate parallel and perpendicular directions with respect to the filler needles, respectively.

Then, we proceeded to the morphological characterization, evaluating the angular, length, and diameter distributions of the conductive chains. This analysis was performed by computing the angle, length, and diameter of the $\mathrm{Fe}_{3} \mathrm{O}_{4} @ \mathrm{Ag}$ chains in SEM images to several zooms $(50-6000 \times)$ and images obtained by optical microscopy using the image processing software ImageJ v1.47. For SEM images, voltage (EHT) $5 \mathrm{kV}$ and extensions of $100 \times\left(3300\right.$ pixels $\left.\cdot \mathrm{cm}^{-1}\right)$ and $300 \times(9800$ pixels $\cdot \mathrm{cm}^{-1}$ ) were typical conditions to compute the average chain length, while to compute chain diameters $3 \mathrm{kV}$ voltages and $4000 \times\left(40\right.$ pixels $\left.\cdot \mu \mathrm{m}^{-1}\right)$ were used.

Figure 5a shows the histogram obtained for the angular distribution of the chains. The histogram is adjusted by a Gaussian distribution function (solid line) centered on the direction of application of the magnetic field during curing $H_{\text {curing }}(\theta=0)$ with standard deviation $\sigma_{\theta}=(4.65 \pm 0.02)^{\circ}$ (count performed on 389 chains). 

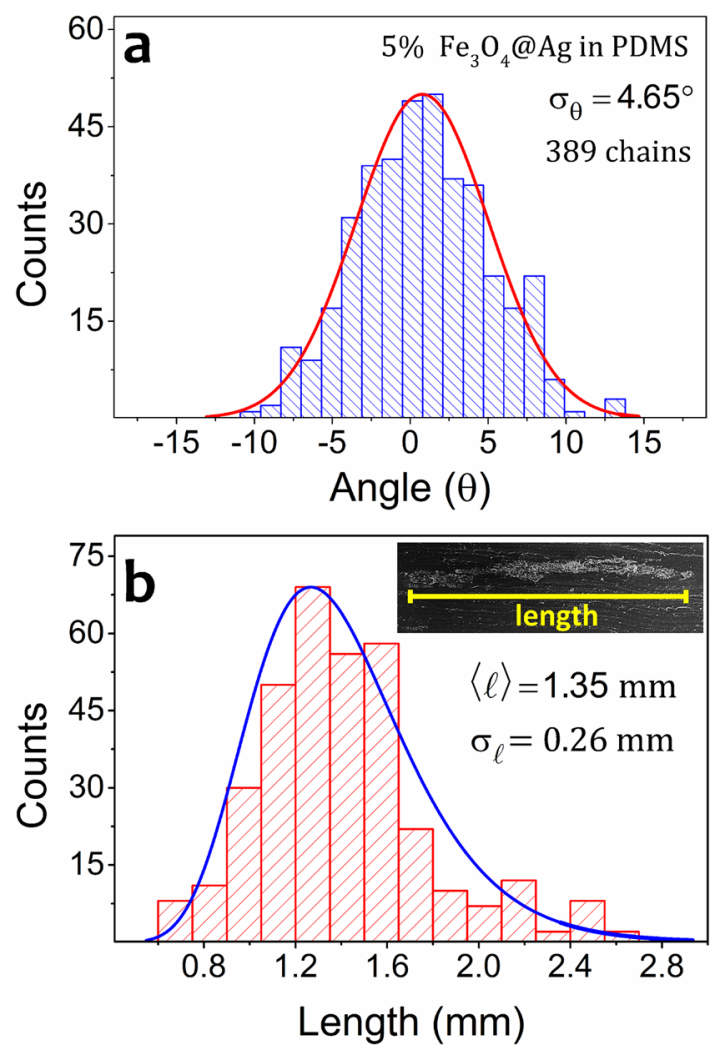

Figure 5. (a) Histogram for the angular distribution of chains in the MRE PDMS $-\mathrm{Fe}_{3} \mathrm{O}_{4} @ \mathrm{Ag} 5 \%$ w/w. The histogram is adjusted by a Gaussian distribution function (solid line). (b) Histogram associated with the distribution of chain lengths, built by measuring the length of 364 chains. The histogram is adjusted by a log-normal distribution function (solid line). Inset: SEM image of a chain.

Figure $5 \mathrm{~b}$ shows the histogram associated with the distribution of chain lengths in the MRE PDMS- $\mathrm{Fe}_{3} \mathrm{O}_{4} @ \mathrm{Ag}$ $5 \% \mathrm{w} / \mathrm{w}$, built by computing 364 chain lengths. It has an excellent degree of adjustment $\left(R^{2}=0.9965\right)$ with the lognormal distribution function

$$
f_{\lambda}=p\left(\lambda ;\langle\lambda\rangle, \sigma_{\lambda}\right)=\frac{1}{\sqrt{2 \pi} \sigma_{\lambda} \lambda} \exp \left(\frac{-[\ln \lambda-\ln \langle\lambda\rangle]^{2}}{2 \sigma_{\lambda}^{2}}\right)
$$

with $\lambda=l$ and fitted parameters $\langle l\rangle=(1.35 \pm 0.01) \mathrm{mm}$ and $\sigma_{l}$ $=(0.26 \pm 0.01) \mathrm{mm}$. The average stick density observed is 11.84 needles $/ \mathrm{mm}^{2}$. Note that here, as we start to study concrete physical systems, we begin to use regular units of length, such as millimeters.

The histogram of values of diameters (not shown) was built from 311 counts and also adjusted by a log-normal distribution (eq 6 with $\lambda=d$ ) with an excellent degree of adjustment, $R^{2}=$ 0.9977 , and parameters $\langle d\rangle=(10.40 \pm 0.02) \mu \mathrm{m}$ and $\sigma_{d}=$ $(0.30 \pm 0.01) \mu \mathrm{m}$. Note that, under the used experimental conditions, the length of the chain is much greater than its diameter, so that the approximation of one-dimensional percolating elements is justified.

\section{SIMULATION OF ANISOTROPIC SYSTEMS}

From the point of view of possible technological applications of MRE, it is important to characterize the degree of anisotropy of the electrical conductivity of a given device. Anisotropy can be introduced essentially in two ways: through an aspect ratio $r \neq$
1 which makes the system asymmetric (relevant when the characteristic length of the percolating objects is not much smaller than the size of the system, as in our study) and through an anisotropic angular distribution of the sticks. These two aspects can be controlled experimentally in systems like the ones discussed in the previous section, and we will now incorporate them in our simulation studies.

The signature of the presence of total anisotropy in the percolation regime will be given by the existence of a percolating cluster in the horizontal direction only, which corresponds to (using the notation introduced in section 3) $\boldsymbol{P}^{\mathrm{HX}}$ taking values very close to 1 . Experimentally, a set of parameters that ensure that condition will constitute what we can call a "safety zone" of total anisotropic percolation and, therefore, of TEA. The main goal of the present work is to establish a methodology and, with it, to arrive at the specification of such a set of parameter values, as an aid to obtaining TEA in devices that demand it (pressure mapping sensors, bidimensional Zebra-like connectors, etc.).

It is important to first determine whether the asymmetry of the box or the anisotropy of the angular distribution contribute equally or not to the global anisotropy of the percolation behavior. In Figure 6 we show the percolation probabilities

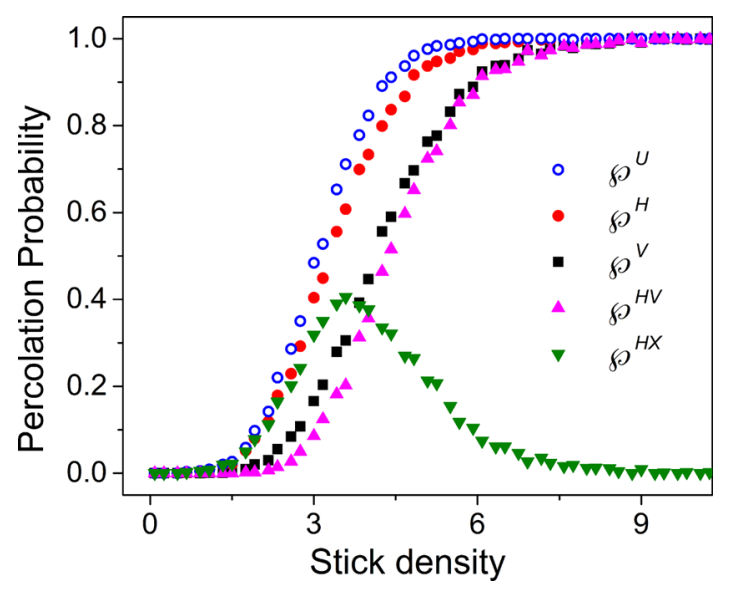

Figure 6. Various percolation probabilities $\left(\boldsymbol{P}^{\mathrm{H}}, \boldsymbol{P}^{\mathrm{V}}, \boldsymbol{P}^{\mathrm{HX}}, \boldsymbol{P}^{\mathrm{U}}, \boldsymbol{P}^{\mathrm{HV}}\right)$ versus density of sticks (chains $/ \mathrm{mm}^{2}$ ) for a rectangular system of aspect ratio $r=L_{x} / L_{y}=3 / 4, L_{x}=3 \mathrm{~mm}$, and isotropic stick distribution with $\langle l\rangle=1.35 \mathrm{~mm}$ and $\sigma_{l}=0.26 \mathrm{~mm}$, for a log-normal distribution of stick lengths. Note that $\boldsymbol{P}^{\mathrm{VX}}$ is not shown since it is always negligible.

$\boldsymbol{P}^{\mathrm{H}}, \boldsymbol{P}^{\mathrm{V}}, \boldsymbol{P}^{\mathrm{HX}}, \boldsymbol{P}^{\mathrm{U}}, \boldsymbol{P}^{\mathrm{HV}}$ for a rectangular isotropic (the stick angular distribution is uniform, with $-\pi \leq \theta \leq \pi$ ) system with aspect ratio $r=3 / 4, L_{x}=3 \mathrm{~mm}$, and log-normal length distribution parameters $\langle l\rangle=1.35 \mathrm{~mm}$ and $\sigma_{l}=0.26 \mathrm{~mm}$ (notice that $P^{\mathrm{VX}}$ is negligible and does not need to be considered in the analysis). We remark that these parameters are taken from an experimental sample, as discussed above (Figure 5). The different percolation probabilities verify the expected inequalities $\boldsymbol{P}^{\mathrm{U}} \geq \boldsymbol{P}^{\mathrm{H}} \geq \boldsymbol{P}^{\mathrm{V}} \geq \boldsymbol{P}^{\mathrm{HV}}$, given the chosen asymmetry of the box. The values of $P^{\mathrm{HX}}$ seen in this figure, never close to unity, indicate that the mere asymmetry of the box $(r \neq 1)$ is not enough to produce a safety zone of totally anisotropic conduction. Therefore, we conclude that in order to achieve effective TEA in bulk or films sample geometries it is required to introduce an internal anisotropy, that is, in the stick angular distribution. This conclusion is consistent with experimental observations. ${ }^{4,12}$ 
We need to introduce a magnitude to characterize in a generic and quantitative way the degree of internal anisotropy of the system of random sticks. Let us denote it macroscopic anisotropy, and it will be given by

$$
\mathcal{A}=\frac{\sum_{j=1}^{N} l_{j}\left|\cos \theta_{j}\right|}{\sum_{j=1}^{N} l_{j}\left|\sin \theta_{j}\right|}
$$

In the limit of infinite percolating objects we have $\mathcal{A}=1$ for isotropic systems, while $\mathcal{A} \rightarrow \infty(0)$ for completely anisotropic systems favoring the horizontal (vertical) direction.

5.1. Influence of $\sigma_{\theta}$. Figure 7 shows examples of random stick systems in a two-dimensional box of sides $L_{x}=3 \mathrm{~mm}$ and
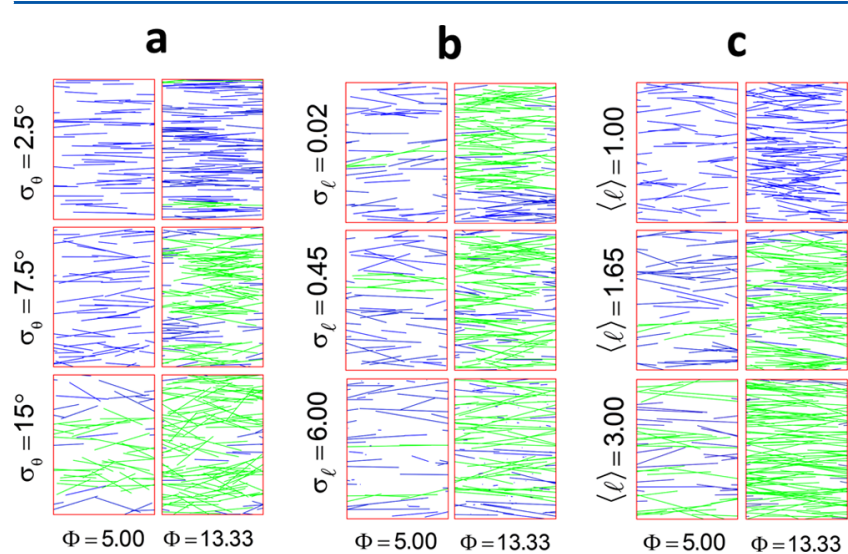

Figure 7. Examples of random stick systems in a two-dimensional box of sides $L_{x}=3 \mathrm{~mm}$ and $L_{y}=4 \mathrm{~mm}$ with anisotropic angular distributions and nonuniform stick length for two different stick densities: $\Phi=5.00$ chains $/ \mathrm{mm}^{2}$ and $\Phi=13.33$ chains $/ \mathrm{mm}^{2}$. The green (color online) sticks belong to a horizontal spanning cluster, and the blue ones do not. The angle distribution is Gaussian centered in zero, and the length distribution is log-normal (as found experimentally). (a) $\langle l\rangle=1.35 \mathrm{~mm}, \sigma_{l}=0.26 \mathrm{~mm}$, (b) $\langle l\rangle=1.35$ $\mathrm{mm}, \sigma_{\theta}=7.5^{\circ}$, (c) $\sigma_{\theta}=7.5^{\circ}, \sigma_{l}=0.26 \mathrm{~mm}$.

$L_{y}=4 \mathrm{~mm}$ (corresponding to the characteristic dimensions of the experimental samples) with anisotropic angular distributions and nonuniform stick length. In all cases the green sticks belong to a horizontal spanning cluster and the blue ones do not. In particular, Figure $7 \mathrm{a}$ shows systems for three different values of the standard deviation of the Gaussian angular distribution, $\sigma_{\theta}$, and two values of the stick density $\Phi$, with parameters of a log-normal distribution $\langle l\rangle=1.35 \mathrm{~mm}$ and $\sigma_{l}=$ $0.26 \mathrm{~mm}$. As expected, for a given value of $\sigma_{\theta}$, the more sticks participating in the spanning cluster the higher the density of sticks. We also note that, for a fixed value of the density $\Phi$, the fraction of sticks that belong to the spanning cluster also increases with $\sigma_{\theta}$.

To evaluate the effect of $\sigma_{\theta},\langle l\rangle$, and $\sigma_{l}$ on the TEA (i.e., on the formation of a spanning cluster only in the horizontal direction), numerical simulations of rectangular systems with $L_{x}$ $=3 \mathrm{~mm}$ and $L_{y}=4 \mathrm{~mm}(r=3 / 4)$ were made, taking the horizontal direction $\left(\theta=0^{\circ}\right)$ as the direction of application of the magnetic field during curing $\left(H_{\text {curing }}\right)$. In particular, to evaluate the effect of $\sigma_{\theta}$, a log-normal distribution for the lengths with parameters $\langle l\rangle=1.35 \mathrm{~mm}$ and $\sigma_{l}=0.26 \mathrm{~mm}$ (empirical parameters for the MRE PDMS- $\mathrm{Fe}_{3} \mathrm{O}_{4} @ \mathrm{Ag} 5 \% \mathrm{w} /$ $\mathrm{w})$, and a Gaussian angular distribution with parameters $\langle\theta\rangle=$ $0^{\circ}$ and different values of standard deviation, $\sigma_{\theta}$, were used in our simulations. Figure 8a shows histograms of macroscopic
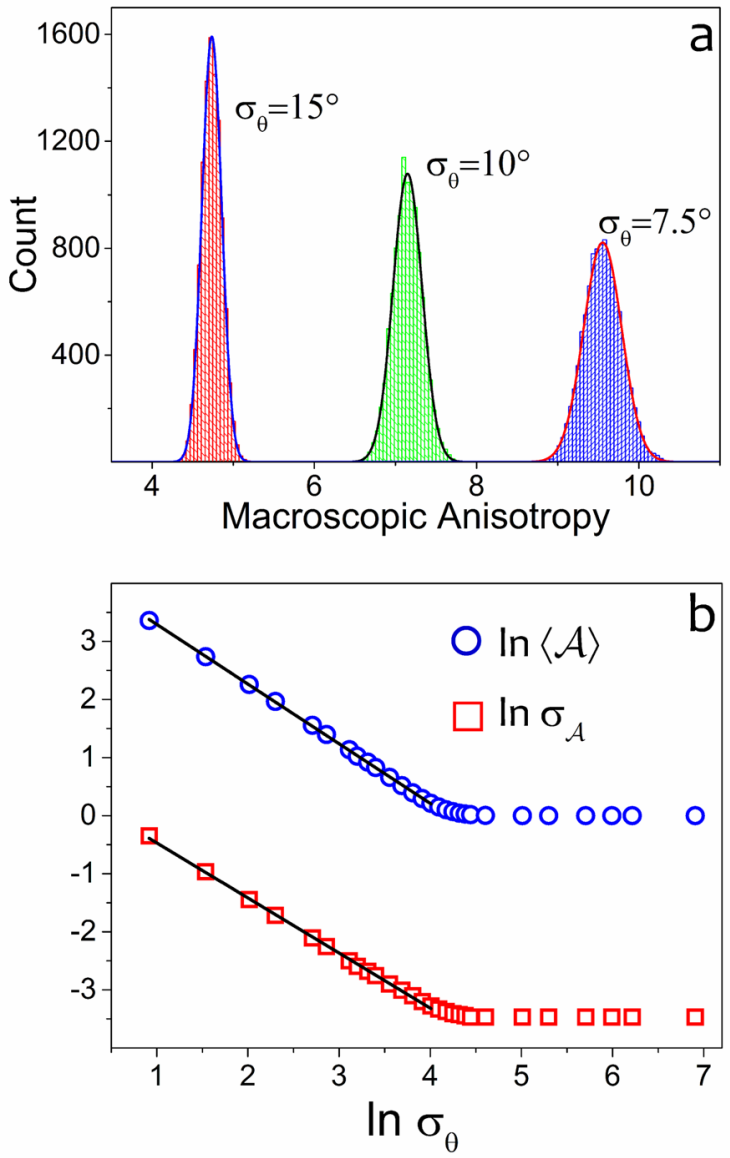

Figure 8. (a) Histograms of the macroscopic anisotropy $\mathcal{A}$ obtained for three different values of $\sigma_{\theta}$ and $N=1000$ for a rectangular system of aspect ratio $r=L_{x} / L_{y}=3 / 4$ and anisotropic stick distribution, with parameters of a log-normal distribution $\langle l\rangle=1.35 \mathrm{~mm}$ and $\sigma_{l}=0.26$ $\mathrm{mm}$. (b) Mean macroscopic anisotropy and its standard deviation versus $\sigma_{\theta}$. For small $\sigma_{\theta}$ we obtain a scaling behavior with exponent approximately equal to -1 for both quantities.

anisotropy, $\mathcal{A}$, obtained for three different values of $\sigma_{\theta}\left(15^{\circ}\right.$, $10^{\circ}$, and $\left.7.5^{\circ}\right)$, each one obtained by performing 10500 repetitions, with $N=1000$. For all values of $\sigma_{\theta}$, the distribution is approximately Gaussian, with an excellent degree of fitting, $R^{2}$ $\geq 0.99926$ (continuous line in Figure 8a). For these distributions, the average macroscopic anisotropy, $\langle\mathcal{A}\rangle$, and its standard deviation, $\sigma_{A}$, follow a monotonously decreasing behavior with $\sigma_{\theta}$, as illustrated in Figure 8b. It is noteworthy that for small values of $\sigma_{\theta}\left(\sigma_{\theta}<55^{\circ}\right)$ there exists a linear relationship between $\ln \langle\mathcal{A}\rangle$ and $\ln \sigma_{\theta}$, as well as between $\ln \sigma_{\mathcal{A}}$ and $\ln \sigma_{\theta}$ (solid lines in Figure 8b, with $R^{2}=0.9998$, slope $=$ $-1.02(7)$, and intercept $=4.32(1)$ for $\ln \langle\mathcal{A}\rangle$, and $R^{2}=0.998$ 97 , slope $=-0.97(6)$, and intercept $=0.48(3)$ for $\left.\ln \sigma_{\mathcal{A}}\right)$.

As described above, a strategy to study the influence of $\sigma_{\theta}$ on the TEA of the composite material is to evaluate curves of $P^{\mathrm{HX}}(\Phi)$ for different values of $\sigma_{\theta}$. Values of $\Phi$ for which $P^{\mathrm{HX}}(\Phi)=1$ (if they exist) constitutes safety zones in terms of TEA: for fixed values of $\langle l\rangle, \sigma_{b}, \sigma_{\theta}$, and densities of percolating objects $\Phi$ in this safety zone, systems are most likely to have TEA, i.e., electrical conductivity only in the horizontal direction by formation of a spanning cluster only in that direction.

Figure $9(\mathrm{a}-\mathrm{c})$ shows the curves of $\boldsymbol{P}^{\mathrm{H}}, \boldsymbol{P}^{\mathrm{V}}$, and $\boldsymbol{P}^{\mathrm{HX}}$, for three values of $\sigma_{\theta}\left(40^{\circ}, 15^{\circ}\right.$, and $\left.4.65^{\circ}\right)$ for systems with log-normal distribution for the stick lengths with parameters $\langle l\rangle=1.35 \mathrm{~mm}$ 
(a)

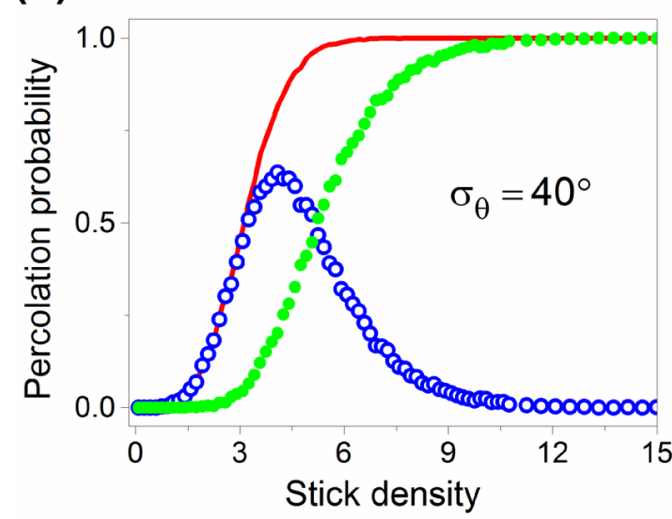

(c)

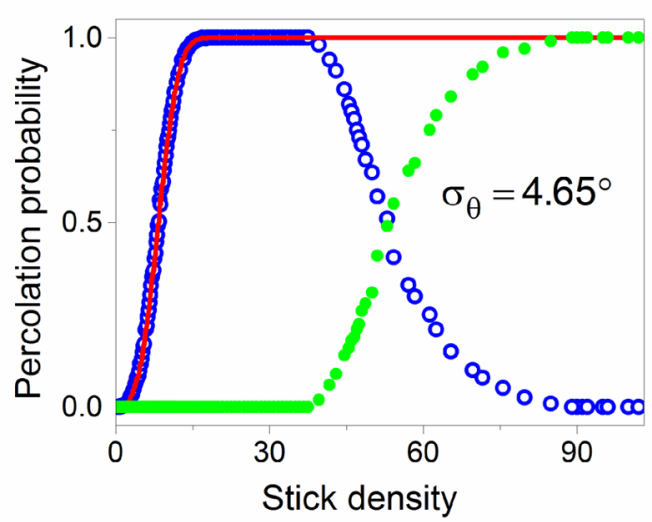

(b)

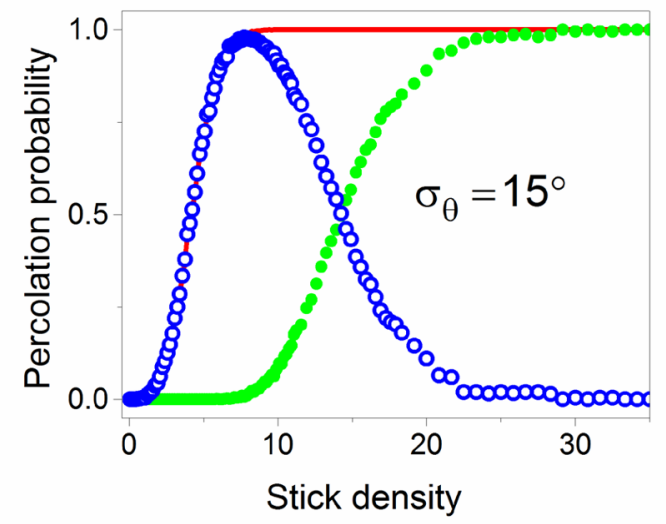

(d)

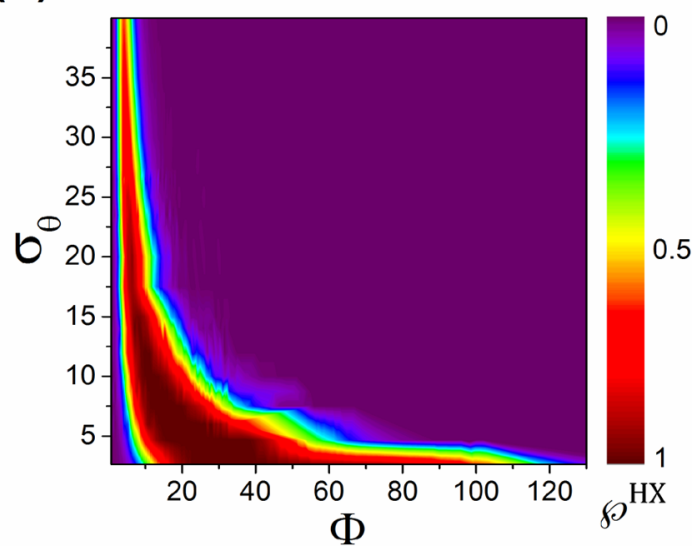

Figure 9. Various percolation probabilities $\left(\boldsymbol{P}^{\mathrm{H}}, \boldsymbol{P}^{\mathrm{V}}, \boldsymbol{P}^{\mathrm{HX}}\right)$ versus density of sticks for a rectangular system of aspect ratio $r=L_{x}=L_{y}=3 / 4$ and anisotropic stick distribution, with parameters of a log-normal distribution $\langle l\rangle=1.35 \mathrm{~mm}$ and $\sigma_{l}=0.26 \mathrm{~mm}$. (a) - (c) Three different values of the standard deviation $\sigma_{\theta}$ of the angular Gaussian distribution. Red solid line: $\boldsymbol{P}^{\mathrm{H}}$, green filled circles: $\boldsymbol{P}^{\mathrm{V}}$, blue open circles: $\boldsymbol{P}^{\mathrm{HX}}$. (d) Probability $\boldsymbol{P}^{\mathrm{HX}}$ as a contour plot versus the stick density $\Phi$ and $\sigma_{\theta}$, showing the full dependence on $\sigma_{\theta}$ not seen in the other panels.

and $\sigma_{l}=0.26 \mathrm{~mm}$. There are values of $\Phi$ for which $P^{\mathrm{HX}}(\Phi)=1$ only when $\sigma_{\theta}<15^{\circ}$. Such behavior of $P^{\mathrm{HX}}(\Phi)$ is detailed in Figure $9 \mathrm{~d}$, which shows the probability $\boldsymbol{P}^{\mathrm{HX}}(\Phi)$ as a contour plot of density versus $\Phi$ and $\sigma_{\theta}$. It can be seen that the range of values of $\Phi$ for which $P^{\mathrm{HX}}(\Phi)=1$ strongly increases with decreasing $\sigma_{\theta}$, and, also, with lower values of the parameter $\sigma_{\theta}$ higher stick density $\Phi$ is required to reach the safety zone.

As described in section 4, all the PDMS- $\mathrm{Fe}_{3} \mathrm{O}_{4} @ \mathrm{Ag} 5 \% \mathrm{w} / \mathrm{w}$ systems synthesized have $\Phi=11.84$ chains $/ \mathrm{mm}^{2}$ and electrical anisotropy (measurable electrical conductivity only in the direction of the magnetic field applied during the curing of the material). Panel c of Figure 9 shows that, for this value of $\Phi$ and the parameters $\sigma_{\theta}=4.65^{\circ},\langle l\rangle=1.35 \mathrm{~mm}$, and $\sigma_{l}=0.26$ $\mathrm{mm}$ (experimental parameters for PDMS $-\mathrm{Fe}_{3} \mathrm{O}_{4} @ \mathrm{Ag} 5 \% \mathrm{w} /$ $\mathrm{w})$, we have a very high only horizontally percolation probability, which shows a very good correlation between our performed simulations and the experimental results obtained.

5.2. Influence of $\sigma_{l}$. Following a similar procedure to the one described in the previous section, in order to evaluate the effect of $\sigma_{b}$ a Gaussian angular distribution with parameters $\langle\theta\rangle$ $=0^{\circ}$ and $\sigma_{\theta}=7.5^{\circ}$ and a log-normal distribution for the lengths with parameters $\langle l\rangle=1.35 \mathrm{~mm}$ and different values of $\sigma_{l}$ were used in our simulations. Figure $7 b$ shows systems for three different values of the standard deviation of the log-normal length distribution, $\sigma_{l}$ and two values of the stick density $\Phi$, with structural parameters $\langle l\rangle=1.35 \mathrm{~mm}$ and $\sigma_{\theta}=7.5^{\circ}$.
Figure 10 shows histograms of macroscopic anisotropy $\mathcal{A}$ obtained for two different values of $\sigma_{l}(0.30$ and $5.00 \mathrm{~mm})$, each one obtained by performing 10500 repetitions, with $N=1000$.
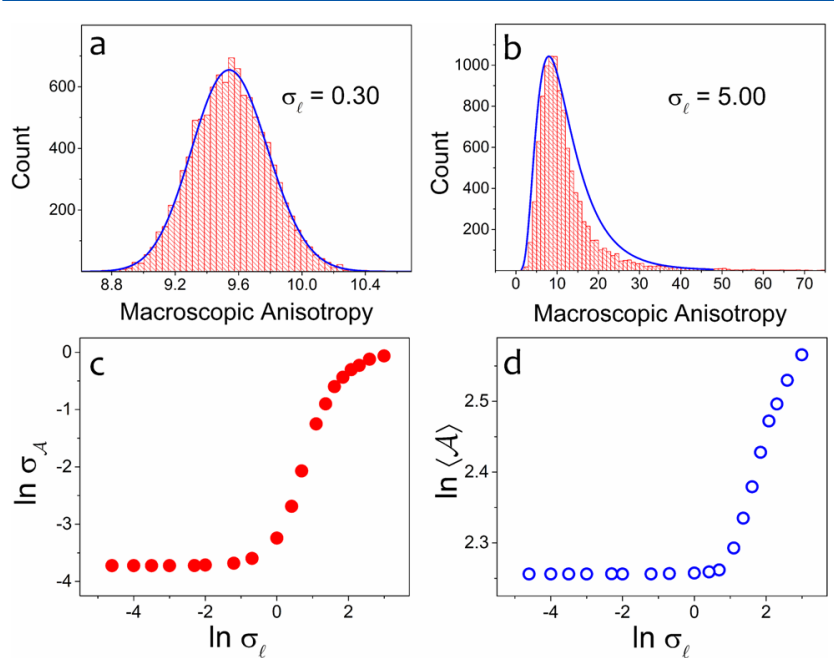

Figure 10. (a,b) Histograms of the macroscopic anisotropy $\mathcal{A}$ obtained for two different values of $\sigma_{l}$ and $N=1000$ for a rectangular system of aspect ratio $r=L_{x} / L_{y}=3 / 4$ and parameters $\langle l\rangle=$ $1.35 \mathrm{~mm}$ and $\sigma_{\theta}=7.5^{\circ}$. (c, d) Macroscopic anisotropy and its standard deviation versus $\sigma_{l}$. 
For all the values of $\sigma_{b}$ the distribution is approximately lognormal, with an excellent degree of adjustment, $R^{2} \geq 0.99511$ (continuous line in Figure $10 \mathrm{a}-\mathrm{b}$ ). At low values of $\sigma_{l}$ the distribution is approximately Gaussian. For these distributions, the average macroscopic anisotropy, $\mathcal{A}$, and its standard deviation, $\sigma_{\mathcal{A}}$, follow a monotonous increasing behavior with $\sigma_{b}$ as illustrated in Figure 10c-d. Again, the strategy that we use to study the influence of $\sigma_{l}$ on the electrical anisotropy of the composite material is to evaluate curves of $P^{\mathrm{HX}}(\Phi)$ for different values of $\sigma_{l}$. Figure $11 \mathrm{~b}$ shows the probability $P^{\mathrm{HX}}(\Phi)$ as a
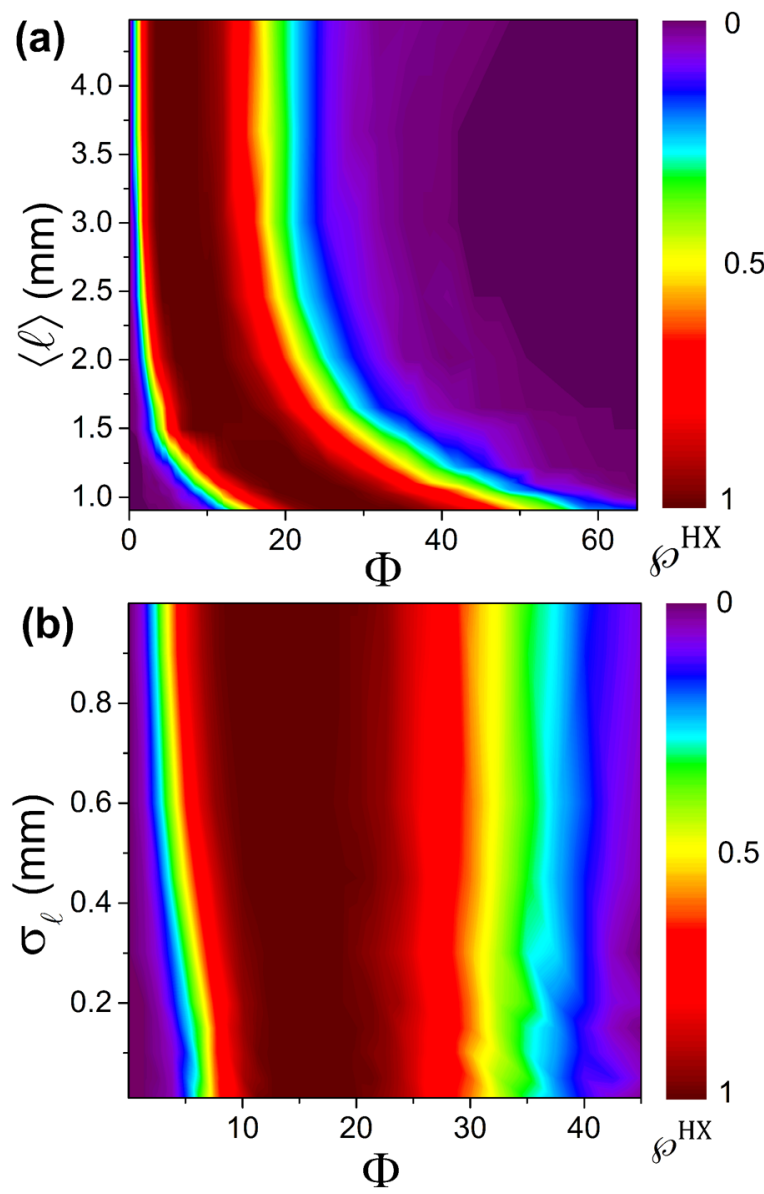

Figure 11. Probability $P^{\mathrm{HX}}$ as a contour plot versus the stick density $\Phi$ and (a) $\langle l\rangle$ with $\sigma_{\theta}=7.5^{\circ}$ and $\sigma_{l}=0.26 \mathrm{~mm}$ and (b) $\sigma_{l}$ with $\langle l\rangle=1.35$ $\mathrm{mm}$ and $\sigma_{\theta}=7.5^{\circ}$.

contour plot versus $\Phi$ and $\sigma_{l}$. It can be seen that the range of values of $\Phi$ for which $P^{\mathrm{HX}}(\Phi) \approx 1$ varies very little with the studied parameter. Only a small increase with increasing $\sigma_{l}$ from 0 to $1 \mathrm{~mm}$ is observed. Above those values (not shown) practically no variation with $\sigma_{l}$ is observed, and therefore the location and size of the safety zone becomes quite insensitive to $\sigma_{l}$.

5.3. Influence of $\langle I\rangle$. In this case, a Gaussian angular distribution with parameters $\langle\theta\rangle=0^{\circ}$ and $\sigma_{\theta}=7.5^{\circ}$ and a lognormal distribution for the lengths with parameters $\sigma_{l}=0.26$ $\mathrm{mm}$ and different values of $\langle l\rangle$ were assumed. Figure 11a shows the probability $P^{\mathrm{HX}}(\Phi)$ as a contour plot versus $\Phi$ and $\langle l\rangle$. It can be seen that the range of values of $\Phi$ for which $P^{\mathrm{HX}}(\Phi) \approx 1$ varies very little with the studied parameter, but the value of $\Phi$ required to reach the safety zone strongly increases with decreasing $\langle l\rangle$. Figure 12 shows a typical macroscopic

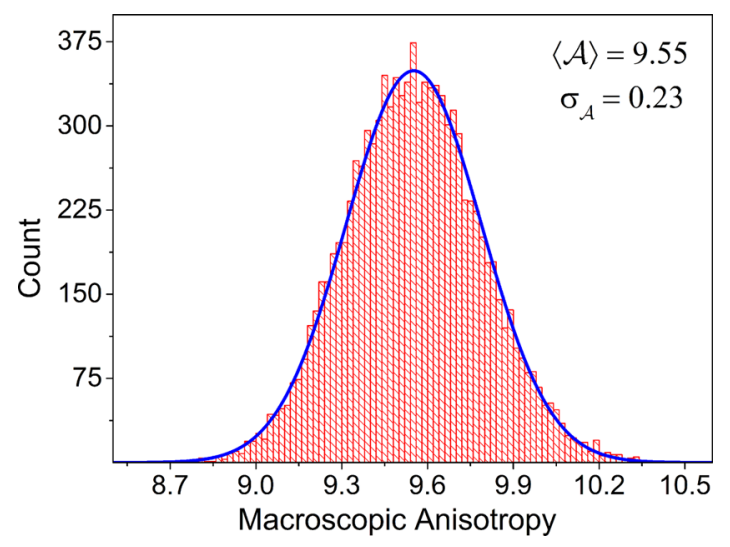

Figure 12. Typical histogram of the macroscopic anisotropy $\mathcal{A}$ obtained for a rectangular system of aspect ratio $r=L_{x} / L_{y}=3 / 4, N=$ 1000 , and parameters $\sigma_{\theta}=7.5^{\circ}$ and $\sigma_{l}=0.26 \mathrm{~mm}$.

anisotropy histogram obtained for $\langle l\rangle=1.22 \mathrm{~mm}$ by performing 10500 repetitions, with $N=1000$. For all the values of $\langle l\rangle$, the distribution is approximately Gaussian, with an excellent degree of adjustment, $R^{2} \geq 0.99977$ (continuous line in Figure 12). Contrary to what was observed for the other two structural parameters, in this case the histograms do not change appreciably for different values of $\langle l\rangle$. For all values of $\langle l\rangle$, the distribution of macroscopic anisotropy is approximately Gaussian with mean values of macroscopic anisotropy $\langle\mathcal{A}\rangle \approx$ 9.55 and standard deviation of macroscopic anisotropy $\sigma_{\mathcal{A}} \approx$ 0.23 .

\section{DISCUSSION AND CONCLUSIONS}

Motivated by experimental work on structured magnetorheologial elastomers, we present a comprehensive study of stick percolation in two-dimensional networks. In order to extract realistic parameters for our simulations, we first carry out a statistical characterization of the distribution of metallic sticks in our previously studied MRE samples. We found that the population of sticks has a log-normal distribution of stick lengths (centered around $1.35 \mathrm{~mm}$ ) and a Gaussian angular distribution. The latter is centered around a preferential axis that is given by the curing magnetic field applied during the sample preparation and has a typical standard deviation of approximately $5^{\circ}$.

In order to simulate the experimental systems, we adopted the model of two-dimensional stick percolation and developed a Monte Carlo numerical algorithm and a computer program implemented in the computer language SAGE. We thoroughly tested our program by reproducing key theoretical results of the known scaling behavior of the percolation probability in square, isotropically distributed systems. Furthermore, we validated numerically for the first time for stick percolation existing theoretical predictions for the different probabilities $P^{\mathrm{HX}}, \boldsymbol{P}^{\mathrm{HV}}$, and $P^{\mathrm{U}}$ in the limit of large system size at the critical percolation density.

We then proceeded to perform extensive numerical simulations of asymmetric (rectangular), anisotropic (in the orientation of the sticks) systems, modeled after the examined experimental samples. A first conclusion is that adopting a rectangular shape with a moderate aspect ratio is by itself not enough to achieve effective TEA. Therefore, it becomes necessary to introduce internal anisotropy, that is, a nonisotropic stick angular distribution, in accordance with 
experimental observations. Consequently, the main objective of the study is then to analyze the effect of key structural parameters of the material, which characterize the angular and length distribution of the sticks (the average length of the sticks $\langle l\rangle$, the standard deviation of the length distribution $\sigma_{l}$ and the standard deviation of the angular distribution $\sigma_{\theta}$ ) on the observation of total electrical anisotropy (TEA). From a practical point of view, TEA is a crucial aspect in the design of nano- or microscale devices like in Zebra-like connectors for parallel flip-chip connections, anisotropic conductive adhesives (ACA), extended pressure-mapping sensors, anisotropic conductive films (ACF), anisotropic conductive paste (ACP), touch screen panels (TSP), and electronic skin (robotic and biomedical applications).

We studied the TEA by computing various probabilities, especially the only horizontal probability percolation function, $\boldsymbol{P}^{\mathrm{HX}}$, and analyzing the macroscopic anisotropy, which quantifies the macroscopic average degree of orientation of the stick population. We find prescriptions to achieve "safe" structural conditions of total electrical anisotropy and thus hope to guide the experimentalist and technologist to choose the experimental conditions needed to make a device with the desired degree of electrical anisotropy. Among other things, we show that there exists a strong dependence of the TEA on the standard deviation of the angular distribution $\left(\sigma_{\theta}\right)$ and on the average length of the sticks, while the standard deviation of the length distribution has little effect. Figure 9 shows clearly this strong dependence on $\sigma_{\theta}$ and presents an interesting re-entrant behavior for a given value of $\sigma_{\theta}{ }^{10}$ which may merit a more exhaustive exploration in the future, in particular for 3D systems. In closing, we remark that the criteria found for TEA explain semiquantitatively the empirical observations made on a fairly large set of samples that we studied experimentally in recent years. This provides a first confirmation of the adequacy of the $2 \mathrm{D}$ stick percolation model to describe structured filmtype magnetorheological elastomers and geometrically analogous systems.

\section{AUTHOR INFORMATION}

\section{Corresponding Author}

*E-mail: pablot@df.uba.ar.

\section{Notes}

The authors declare no competing financial interest.

\section{ACKNOWLEDGMENTS}

We thank Cristopher Moore and Stephan Mertens for useful comments. We also thank the SAGE users community for fruitful discussions and Prof. William Stein (University of Washington) for allowing us to run some of our simulations on SageMathCloud. R.M.N. and P.I.T. are research members of the National Council of Research and Technology (CONICET, Argentina). Financial support was received from UBA (UBACyT projects 2012-2015, number 20020110100098 and 2011-2014 number 20020100100741), and from the Ministry of Science, Technology, and Innovation (MINCYT-FONCYT, Argentina, PICT 2011-0377). Support from the Center of Documental Production (CePro) and the Center of Advanced Microscopy (CMA) (FCEyN, UBA) to obtain the shown SEMTEM images and from the Low-Temperatures Laboratory (Department of Physics, FCEyN, UBA) to implement the device used to generate the structured materials is gratefully acknowledged.

\section{REFERENCES}

(1) Gupta, M. P.; Behnam, A.; Lian, F.; Estrada, D.; Pop, E.; Kumar, S. High Field Breakdown Characteristics of Carbon Nanotube Thin Film Transistors. Nanotechnology 2013, 24, 405204.

(2) Zeng, X.; Xu, X.; Shenai, P. M.; Kovalev, E.; Baudot, C.; Mathews, N.; Zhao, Y. Characteristics of the Electrical Percolation in Carbon Nanotubes/Polymer Nanocomposites. J. Phys. Chem. C 2011, $115,21685-21690$.

(3) Kocabas, C.; Meitl, M. A.; Gaur, A.; Shim, M.; Rogers, J. A. Aligned Arrays of Single-Walled Carbon Nanotubes Generated from Random Networks by Orientationally Selective Laser Ablation. Nano Lett. 2004, 4, 2421-2426.

(4) Mietta, J. L.; Jorge, G.; Perez, O. E.; Maeder, T.; Negri, R. M. Superparamagnetic Anisotropic Elastomer Connectors Exhibiting Reversible Magneto-Piezoresistivity. Sens. Actuators Phys. 2013, 192, 34-41.

(5) Snow, E. S.; Campbell, P. M.; Ancona, M. G.; Novak, J. P. HighMobility Carbon-Nanotube Thin-Film Transistors on a Polymeric Substrate. Appl. Phys. Lett. 2005, 86, 033105.

(6) Mietta, J. L.; Jorge, G. A.; Negri, R. M. A Flexible Strain Gauge Exhibiting Reversible Piezoresistivity Based on an Anisotropic Magnetorheological Polymer. Smart Mater. Struct. 2014, 23, 085026.

(7) Collins, P. G.; Bradley, K.; Ishigami, M.; Zettl, A. Extreme Oxygen Sensitivity of Electronic Properties of Carbon Nanotubes. Science 2000, 287, 1801-1804.

(8) Hicks, J.; Behnam, A.; Ural, A. Resistivity in Percolation Networks of One-Dimensional Elements with a Length Distribution. Phys. Rev. E 2009, 79, 012102

(9) Pasquier, A. D.; Unalan, H. E.; Kanwal, A.; Miller, S.; Chhowalla, M. Conducting and Transparent Single-Wall Carbon Nanotube Electrodes for Polymer-Fullerene Solar Cells. Appl. Phys. Lett. 2005, 87, 203511.

(10) Du, F.; Fischer, J. E.; Winey, K. I. Effect of Nanotube Alignment on Percolation Conductivity in Carbon Nanotube/Polymer Composites. Phys. Rev. B 2005, 72, 121404(R).

(11) Peng, H.; Sun, X. Highly Aligned Carbon Nanotube/Polymer Composites with Much Improved Electrical Conductivities. Chem. Phys. Lett. 2009, 471, 103-105.

(12) Mietta, J. L.; Ruiz, M. M.; Antonel, P. S.; Perez, O. E.; Butera, A.; Jorge, G.; Negri, R. M. Anisotropic Magnetoresistance and Piezoresistivity in Structured $\mathrm{Fe}_{3} \mathrm{O}_{4}-$ Silver Particles in PDMS Elastomers at Room Temperature. Langmuir 2012, 28, 6985-6996.

(13) Antonel, P. S.; Jorge, G.; Perez, O. E.; Butera, A.; Leyva, A. G.; Negri, R. M. Magnetic and Elastic Properties of $\mathrm{CoFe}_{2} \mathrm{O}_{4}-$ Poly(dimethylsiloxane) Magnetically Oriented Elastomer Nanocomposites. J. Appl. Phys. 2011, 110, 043920.

(14) Kchit, N.; Bossis, G. Piezoresistivity of Magnetorheological Elastomers. J. Phys.: Condens. Matter 2008, 20, 204136.

(15) Boczkowska, A.; Awietj, S. Microstructure and Properties of Magnetorheological Elastomers; InTech: Rijeka, Croatia, 2012.

(16) Yook, S.-H.; Choi, W.; Kim, Y. Conductivity of Stick Percolation Clusters with Anisotropic Alignments. J. Korean Phys. Soc. 2012, 61, 1257-1262.

(17) Li, J.; Zhang, S.-L. Finite-Size Scaling in Stick Percolation. Phys. Rev. E 2009, 80, 040104(R).

(18) Kim, Y.; Yun, Y.; Yook, S.-H. Explosive Percolation in a Nanotube-Based System. Phys. Rev. E 2010, 82, 061105.

(19) Žeželj, M.; Stanković, I.; Belić, A. Finite-Size Scaling in Asymmetric Systems of Percolating Sticks. Phys. Rev. E 2012, 85, 021101 .

(20) Stauffer, D.; Aharony, A. Introduction to Percolation Theory; Taylor \& Francis: Oxford, U.K., 1992.

(21) Matoz-Fernández, D. A.; Linares, D. H.; Ramírez-Pastor, A. J. Nonmonotonic Size Dependence of the Critical Concentration in 2D Percolation of Straight Rigid Rods under Equilibrium Conditions. Eur. Phys. J. B 2012, 85, 296.

(22) Ambrosetti, G.; Grimaldi, C.; Balberg, I.; Maeder, T.; Danani, A.; Ryser, P. Solution of the Tunneling-Percolation Problem in the Nanocomposite Regime. Phys. Rev. B 2010, 81, 155434. 
(23) Nigro, B.; Grimaldi, C.; Ryser, P.; Chatterjee, A. P.; van der Schoot, P. Quasiuniversal Connectedness Percolation of Polydisperse Rod Systems. Phys. Rev. Lett. 2013, 110, 015701.

(24) Lin, K. C.; Lee, D.; An, L.; Joo, Y. H. Finite-Size Scaling Features of Electric Conductivity Percolation in Nanocomposites. Nanosci. Nanoeng. 2013, 1, 15-22.

(25) Zheng, X.; Forest, M. G.; Vaia, R. A.; Arlen, M.; Zhou, R. A Strategy for Dimensional Percolation in Sheared Nanorod Dispersions. Adv. Mater. 2007, 19, 4038-4043.

(26) White, S. I.; DiDonna, B. A.; Mu, M.; Lubensky, T. C.; Winey, K. I. Simulations and Electrical Conductivity of Percolated Networks of Finite Rods Various Degrees of Axial Alignment. Phys. Rev. B 2009, 79, 024301.

(27) Mertens, S.; Moore, C. Continuum Percolation Thresholds in Two Dimensions. Phys. Rev. E 2012, 86, 061109.

(28) Newman, M. E. J.; Ziff, R. M. Fast Monte Carlo Algorithm for Site or Bond Percolation. Phys. Rev. E 2001, 64, 016706.

(29) de Berg, M.; Cheong, O.; van Kreveld, M. Computational Geometry: Algorithms and Applications; Springer: New York, 2008.

(30) Erciyes, K. Distributed Graph Algorithms for Computer Networks; Springer: New York, 2013.

(31) Devore, J. L. Probability and Statistics for Engineering and the Sciences; Cengage Learning, 2012.

(32) Hovi, J.-P.; Aharony, A. Scaling and Universality in the Spanning Probability for Percolation. Phys. Rev. E 1996, 53, 235-253.

(33) Ziff, R. M. Correction-to-Scaling Exponent for Two-Dimensional Percolation. Phys. Rev. E 2011, 83, 020107.

(34) Stauffer, D. Search for Logarithmic Factors near the TwoDimensional Percolation Threshold. Phys. Lett. A 1981, 83, 404-405.

(35) Ziff, R. M.; Newman, M. E. J. Convergence of Threshold Estimates for Two-Dimensional Percolation. Phys. Rev. E 2002, 66, 016129.

(36) Rintoul, M. D.; Torquato, S. Precise Determination of the Critical Threshold and Exponents in a Three-Dimensional Continuum Percolation Model. J. Phys. A: Math. Gen.. 1997, 30, 585-592.

(37) Cardy, J. The Number of Incipient Spanning Clusters in TwoDimensional Percolation. J. Phys. A: Math. Gen. 1998, 29, L105-L110.

(38) Watts, G. M. T. A Crossing Probability for Critical Percolation in Two Dimensions. J. Phys. A: Math. Gen. 1996, 29, L363-L368.

(39) Langlands, R.; Pouliot, P.; Saint-Aubin, Y. Conformal Invariance in Two-Dimensional Percolation. Bull. Am. Math. Soc. 1994, 30, 1-61.

(40) Pruessner, G.; Moloney, N. R. Numerical Results for Crossing, Spanning and Wrapping in Two-Dimensional Percolation. J. Phys. A: Math. Gen. 2003, 36, 11213.

(41) Sheffield, S.; Wilson, D. B. Schramm's Proof of Watts' Formula. Ann. Probab. 2011, 39, 1844-1863.

(42) Godoy, M.; Moreno, A. J.; Jorge, G. A.; Ferrari, H. J.; Antonel, P. S.; Mietta, J. L.; Ruiz, M.; Negri, R. M.; Pettinari, M. J.; Bekeris, V. Micrometric Periodic Assembly of Magnetotactic Bacteria and Magnetic Nanoparticles Using Audio Tapes. J. Appl. Phys. 2012, 111, 044905.

(43) Butera, A.; Álvarez, N.; Jorge, G.; Ruiz, M. M.; Mietta, J. L.; Negri, R. M. Microwave Response of Anisotropic Magnetorheological Elastomers: Model and Experiments. Phys. Rev. B 2012, 86, 144424.

(44) Efimenko, K.; Wallace, W. E.; Genzer, J. Surface Modification of Sylgard-184 Poly(dimethylsiloxane) Networks by Ultraviolet and Ultraviolet/Ozone Treatment. J. Colloid Interface Sci. 2002, 254, $306-315$.

(45) Esteves, A. C. C.; Brokken-Zijp, J.; Laven, J.; Huinink, H. P.; Reuvers, N. J. W.; Van, M. P.; de With, G. Influence of Cross-Linker Concentration on the Cross-Linking of PDMS and the Network Structures Formed. Polymer 2009, 50, 3955-3966. 